\title{
Exome sequencing identified rare variants in genes HSPG2 and ATP2B4 in a family segregating developmental dysplasia of the hip
}

\author{
Sulman Basit ${ }^{1 *}$, Alia M. Albalawi ${ }^{1}$, Essa Alharby ${ }^{1}$ and Khalid I. Khoshhal ${ }^{2}$
}

\begin{abstract}
Background: Developmental dysplasia of the hip $(\mathrm{DDH})$ is a common pathological condition of the musculoskeletal system in infants which results in a congenital and developmental malformation of the hip joint. $\mathrm{DDH}$ is a spectrum of pathologies affecting the infant hip ranging from asymptomatic subtle radiographic signs through mild instability to frank dislocations with acetabular dysplasia. A Saudi family with three affected individuals with DDH was identified and genetic analysis was performed to detect the possible genetic defect(s) underlying DDH in the affected members of the family.

Methods: We performed whole genome genotyping using Illumina HumanOmni $2.5 \mathrm{M}$ array and whole exome sequencing (WES) using Nextera Rapid capture kit and Illumina NextSeq500 instrument in four individuals of a family with DDH.

Results: SNP data analysis did not identify any runs of homozygosity and copy number variations. Identity-bydescent (IBD) analysis on whole genome genotyping data identified a shared haplotypes on chromosome 1 in affected individuals. An analysis of the WES data identified rare heterozygous variants in HSPG2 and ATP2B4 genes in the affected individuals. Multiple prediction software predicted that the variants identified are damaging. Moreover, in silico analysis showed that HSPG2 regulates ATP2B4 expression using a variety of transcription factors.

Conclusion: Our results indicate that there might be a functional epistatic interaction between HSPG2 and ATP2B4, and DDH in the family studied is due to a combined effect of both variants. These variants are also present in the asymptomatic mother suggesting that the variants in HSPG2 and ATP2B4 are incompletely penetrant. This study provides the first evidence of digenic inheritance of DDH in a family and extends the spectrum of genetic heterogeneity in this human disorder.
\end{abstract}

Keywords: ATP2B4, Developmental dysplasia of the hip, Exome sequencing, Perlecan, HSPG2

\section{Background}

Developmental dysplasia of the hip (DDH; MIM 142700) is a developmental disorder of the hip joint that results in an abnormal socket of the femoral head, ranging from instability, subluxation and complete dislocation of the hip joint, reduced joint function, and accelerated wear of the articular surface, which may result in early arthritis

\footnotetext{
* Correspondence: sbasit.phd@gmail.com

${ }^{1}$ Centre for Genetics and Inherited Diseases, Taibah University, Almadinah

Almunawwarah 30001, Saudi Arabia

Full list of author information is available at the end of the article
}

$[1,2]$. Phenotypic variability has been observed in individuals affected with DDH.

The etiology of DDH is multifactorial, involving both genetic and environmental risk factors. Non-genetic risk factors include breech presentation, oligohydramnios, and primiparity [3]. Families with members affected with DDH has been reported [4-8]. This provides supports to the argument that DDH has a strong genetic basis [9-12]. Linkage analysis, exome sequencing and case-control association studies have so far identified several loci/genes associated with DDH [13]. Although, DDH is observed as 
an isolated condition in majority of patients with unilateral or bilateral hip involvement, it can be associated with other conditions such as clubfeet, renal malformations and cardiac anomalies [14].

An incidence of 1.5 - 20 cases of DDH per 1000 live births has been described [15]. Though, in some countries including Italy, Japan and other Mediterranean countries the prevalence of DDH is higher [16]. The variations in incidence rate is due to differences in diagnostic methods and the timing of evaluation of DDH affected individuals [17]. In the Middle East, few hospital based studies have shown an incidence rate of 3.17 - 3.50 per 1000 live births [18-20].

Genetic risk factors for DDH have mainly been searched for through case-control studies which have identified several candidate genes associated with bone and joint biology $[4,6,8,20-24]$. These includes GDF5, TBX4, ASPN, TGFB1 and IL6 genes. However, no firm genotypephenotype relations have been established for DDH yet.

Linkage analysis based on whole genome genotyping data has detected several chromosomal loci linked with DDH phenotype in extended pedigrees $[6,8,20]$. However, until now, studies presenting results from genetic analysis of such large families segregating DDH are very limited. So far, only two genes of interest were identified from such analyses. Feldman et al [2] identified a variant in CX3CR1 shared by all DDH affected members of a 4-generational family. More recently, a mutation in the gene UFSP2 was found to follow an autosomal dominant inheritance pattern with reduced penetrance (estimated at $80 \%$ ) in a family from South-Africa with Buekes hip dysplasia [25].

The paucity of genetic information on DDH patients from Middle East emphasizes the need for comprehensive genomic studies to identify the candidate gene(s) in this population. We performed whole genome single nucleotide polymorphism (SNP) genotyping and whole exome sequencing (WES) in a Saudi family with three individuals showing DDH. We identified probably pathogenic heterozygous variants in HSPG2 and ATP2B4 genes in the affected individuals. Our data showed that the genetic variants underlying DDH are not completely penetrant.

\section{Methods}

Genetic analysis of 5 individuals (III:3, III:4, III:5, IV:1, IV:2) including 3 affected members (III:5, IV:1, IV:2) in a single family of Saudi origin (Fig. 1a) showing an isolated form of DDH was carried out. All affected individuals in the family were clinically and radiologically examined by a Pediatric Orthopedic Surgeon. Permission to undertake the study was obtained from the Research Ethics Committee (REC) of Taibah University. An informed consent for genetic evaluation was obtained from all participants. The blood samples from 5 individuals were obtained in EDTA tubes. Genomic DNA was isolated using QIAamp DNA mini kit. Concentration and quality of DNA was assessed by Nanodrop spectrophotometer (Green BioResearch, Baton Rouge, LA 70808, USA) and Qubit fluorometer (ThermoFisher Scientific Inc).

\section{Sanger Sequencing of Known Genes Associated with DDH} The family members were first tested for mutations in the known genes previously associated with DDH. The genes sequenced included asporin $(A S P N)$ at chromosome 9q22.31, chemokine receptor 1 (CX3CR1) at chromosome 3p22.2, dickkopf WNT signaling pathway inhibitor 1 (DKK1) at chromosome 10q21.2, growth differentiation factor 5 (GDF5) at chromosome 20q11.22, homeobox B9 (HOXB9) at chromosome 17q21.32, homeobox D9 (HOXD9) at chromosome 2q31.1, pappalysin 2 (PAPPA2)

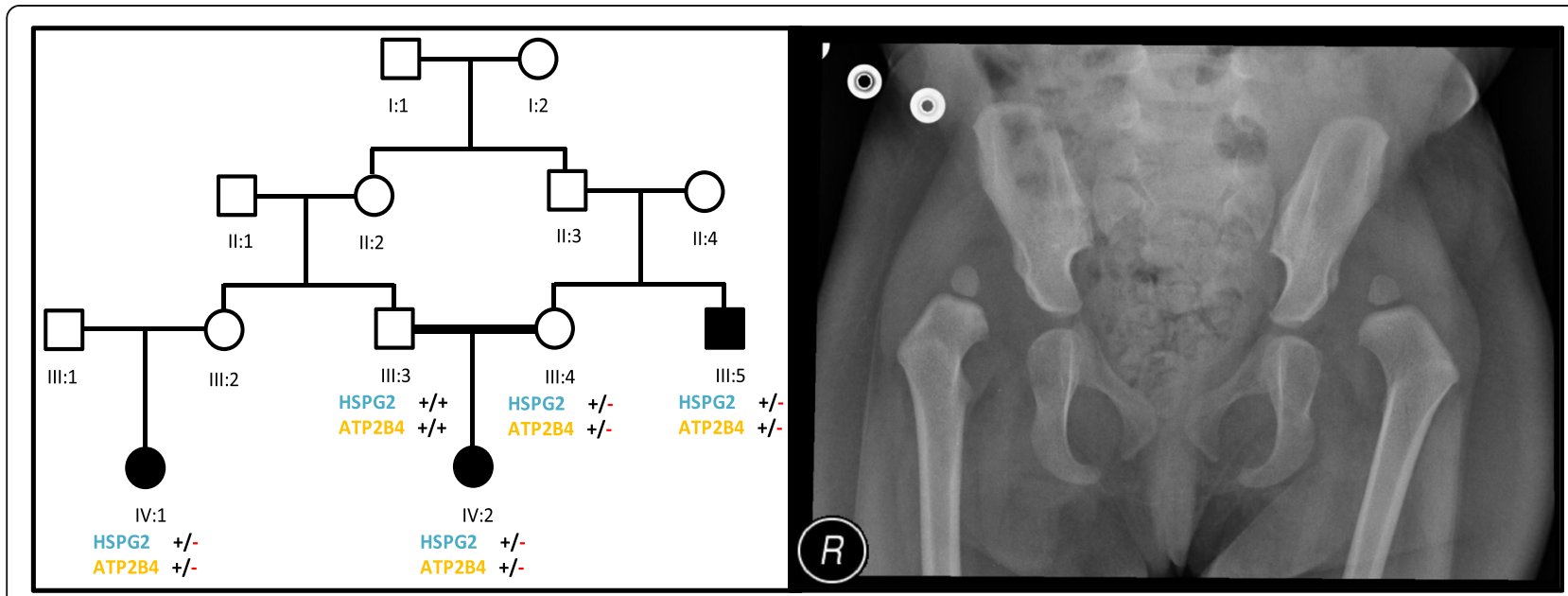

Fig. 1 Pedigree drawings of the family with Developmental Dysplasia of the Hip (DDH). Open squares and circles represent unaffected males and females, respectively. Filled squares and circles represent affected individuals. Double lines between symbols are representative of consanguineous unions. +/+ indicate wild type homozygote, while $+/$ - indicate heterozygotes (a). Anterio-posterior radiographs of the pelvis of an affected individual (IV:2) showing bilateral hip dislocation with bilateral acetabular dysplasia (b) 
at chromosome 1q25.2, and transforming growth factor, beta 1 (TGFB1) at chromosome 19q13.2.

\section{Whole genome SNP genotyping}

Illumina HumanOmni 2.5 M SNP array was used for whole genome SNP genotyping. A total of $200 \mathrm{ng}$ genomic DNA from four members (III:3, III:4, III:5, IV:2) of a family was used as a starting material. Briefly, $0.1 \mathrm{~N} \mathrm{NaOH}$ was used for DNA denaturation and whole genome was amplified out with Random Primers Mix (RPM) using Multi Sample Master Mix (MSM). Enzymatic fragmentation of the amplified DNA was carried out using Fragmentation Mix (FMS) followed by precipitation using Precipitation Mix 1 (PM1) and 2-propanol. Fragmented DNA was hybridized to BeadChip by denaturing the sample and dispensing $35 \mathrm{ul}$ of the sample onto the BeadChip section followed by incubation for 18 hours at $48{ }^{\circ} \mathrm{C}$ in the hybridization oven. Single base extension on BeadChips was performed after washing and staining. Single base extension reaction incorporates labeled nucleotides into the extended primers. Scanning of BeadChips was performed in Illumina iScan using iScan control software. Illumina GenomeStudio software and HomozygosityMapper were used to call loss of heterozygous (LOH) regions while the Illumina Genome Viewer incorporated in GenomeStudio was used to detect copy number variations (CNVs) in the genome. Identity-by-descent (IBD) analysis was carried out using PLINK [26] to identify shared genomic regions.

\section{Whole exome sequencing (WES)}

Four individuals (III:3, III:4, III:5, IV:2) were initially available for exome sequencing. Nextera Rapid Capture Exome kit was used for library preparation and exome enrichment. This kit captures 214,405 exons and splice site and covers 98.3\% RefSeq. Cluster generation and DNA sequencing was performed on Illumina NextSeq500 instrument.

Briefly, $50 \mathrm{ng}$ of DNA was fragmented enzymatically and tagged with adaptor sequences (tagmentation) followed by purification and amplification of the purified tagmented DNA. Resulting libraries were purified with magnetic beads and target regions were captured with whole exome oligos followed by PCR amplification of the enriched library. Quantification of enriched library was performed with Qubit fluorometer and library size distribution was measured with Agilent Bioanalyzer. Quantified DNA library was loaded on flow cell for subsequent cluster generation and sequencing was carried out with an Illumina NextSeq500 instrument. NextSeq500 generates bcl files for each of the four lanes. These files were converted to fastq files using BCL2FASTQ tool. BWA aligner incorporated in BaseSpace was used to align fastq files to the reference genome using the BWA-MEM algorithm. Variants were called using genome analysis tool kit (GATK). Illumina VariantStudio was used for annotation and filtration of the genomic variants
(Additional file 1: Figure S1). Loss of heterozygosity (LOH) analysis was performed on exome data of individuals III:5 and IV:2.

Sanger sequencing was performed for variants of interest to confirm the variants discovered by WES. Genomic sequences of the HSPG2 and ATP2B4 genes, including exons, introns, $5^{\prime}$ untranslated region and $3^{\prime}$ untranslated region, were retrieved from the Ensembl genome browser (http://asia.ensembl.org/index.html). Primer-3 software (http://frodo.wi.mit.edu/primer3/) was used to design primers for PCR amplification of the variants and their flanking regions. BIOEDIT sequence alignment editor version 6.0.7 (Ibis Biosciences Inc., Carlsbad, CA, USA) was used for sequence alignment.

Sanger reads for known genes sequenced in this study, whole genome SNP genotypes and exome variant files are available upon request.

\section{Results}

\section{Clinical description of patients}

The manifestation of DDH is bilateral hip dislocation in both affected individuals. Proband (IV:2) is a 2 year old female with bilateral DDH, discovered after watching her gait during walking. Her pelvic radiograph showed bilateral hip dislocation with bilateral acetabular dysplasia (Fig. 1b). She was treated by staged bilateral open hip reduction and pelvic osteotomy.

Her 30 years old maternal uncle (III:5) was presented with the same phenotypic manifestation. A third affected individual (IV:1) was identified while interviewing elders of the family. All affected individuals are of normal stature and have no other associated abnormality.

\section{Whole genome genotyping data analysis}

Sequencing data analysis of the known genes ( $A S P N$, CX3CR1, DKK1, GDF5, HOXB9, HOXD9, PAPPA2, TGFB1) excluded their involvement in causing DDH in the present family.

After excluding known genes, whole genome homozygosity mapping was carried out using Illumina $2.5 \mathrm{M}$ BeadChip array. SNP genotypes were called by the BRLMM algorithm incorporated in Illumina GenomeStudio genotyping module. A call rate of more than $99 \%$ was obtained across the entire sample. Mapping order and physical and genetic distances of SNPs were obtained from Illumina. Analysis of SNP data to detect LOH was conducted using GenomeStudio software, HomozygosityMapper and AutoSNPa. However, no shared blocks of homozygosity were found in affected individuals. Moreover, Genome Viewer failed to find any shared pathogenic CNVs in the affected individuals. In order to identify genomic regions that are shared between affected individuals, genotyping data was subjected to IBD analysis using PLINK [26]. A shared haplotypes were detected on chromosome 1 in all 3 affected 
individuals (Fig. 2). The percentage of homozygosity ranged from $7-29 \%$ based on regions of homozygosity $>10 \mathrm{Mb}$.

\section{Whole exome sequencing (WES) data analysis}

WES was performed in the four individuals including the two affected (III:5, IV:2) and the two asymptomatic members (III:3, III:4) of the family. Average coverage achieved was between $65-92 \%$ (Table 1 and Table 2). The resulting variant call format (VCF) files contains on average 80,000 variants. These variants were filtered based on quality, frequency, genomic position, protein effect, pathogenicity and previous associations with the phenotype (Additional file 2: Figure S2).
$\mathrm{LOH}$ analysis was performed on exome data of individuals III:5 and IV:2. However, no shared blocks of homozygosity were detected for these samples. In a first approach to find possible relevant variants in the affected members of this family, we looked for the previously reported variants in the CX3CR1 and UFSP2 genes; and then expanded the search for other potential candidate variants in the same genes. However, the described variants were not present in any of the family members nor any other candidate variants were found in these genes. Furthermore, WES reads for already known genes including ASPN, CX3CR1, DKK1, GDF5, HOXB9, HOXD9, PAPPA2, $T G F B 1$ were aligned and inspected manually for any

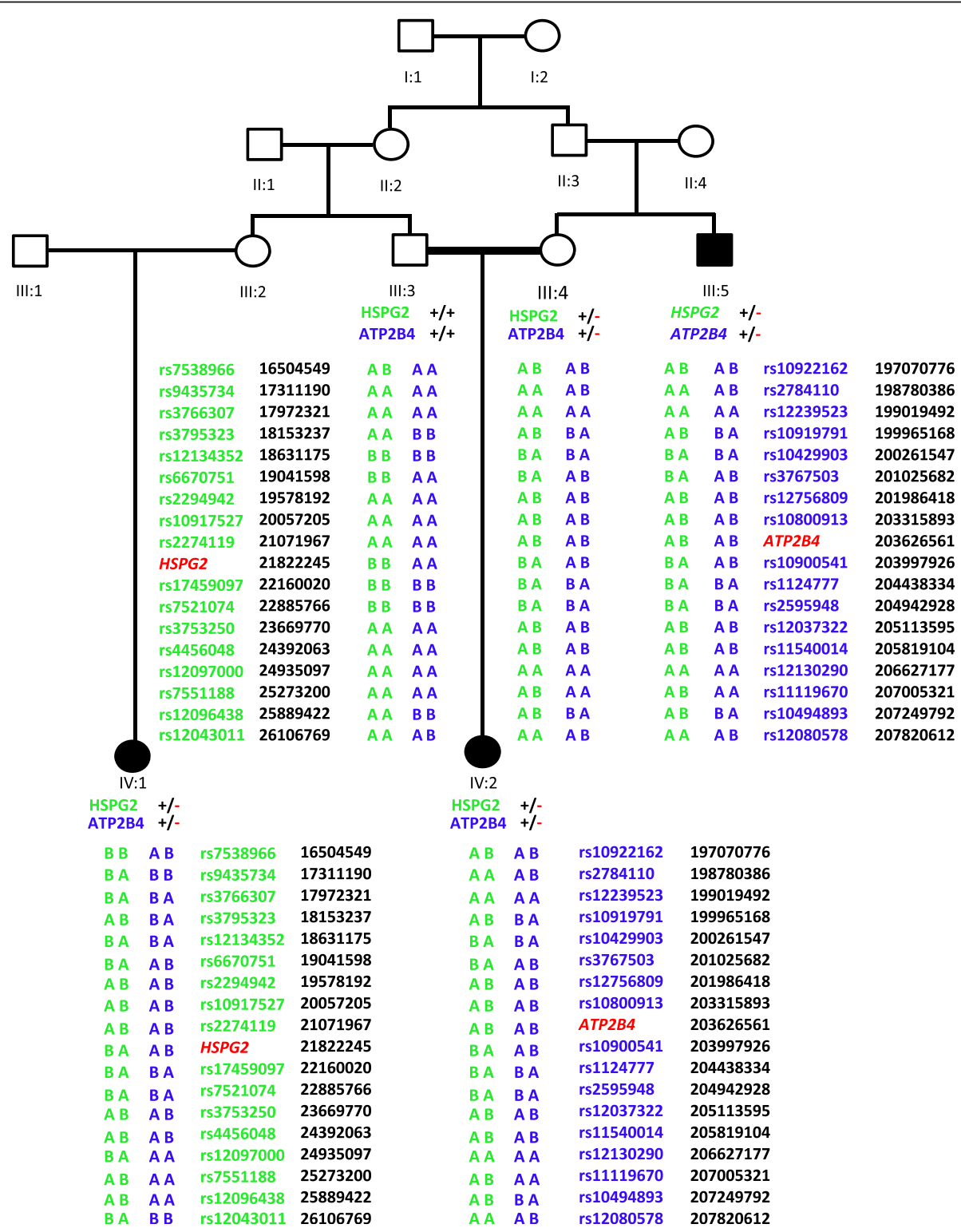

Fig. 2 Haplotypes obtained from PLINK based IBD analysis using SNP genotyping data. All three affected individuals share haplotype carrying the mutant allele. Green color is used for HSPG2 flanking haplotypes while blue color is used for ATP2B4 flanking haplotypes 
Table 1 Pre-alignment statistics

\begin{tabular}{lllcccccc}
\hline Sample ID & $\begin{array}{l}\text { Total Read } \\
\text { Bases (bp) }\end{array}$ & Total Reads & Average read length & GC (\%) & Q20 (\%) & Q30 (\%) & $\begin{array}{l}\text { Mappable } \\
\text { Reads (\%) }\end{array}$ & $\begin{array}{l}\text { Mean depth of } \\
\text { target region (X) }\end{array}$ \\
\hline III-3 & $11,149,473,250$ & $84,775,682$ & 131.52 & 44.7 & 78.0 & 64.3 & 95.6 & 65.0 \\
III-4 & $15,035,517,174$ & $107,770,286$ & 139.51 & 44.0 & 77.6 & 63.6 & 95.8 & 79.2 \\
III-5 & $15,498,839,720$ & $113,685,720$ & 136.33 & 44.1 & 81.2 & 68.0 & 96.7 & 92.8 \\
IV-2 & $12,988,837,852$ & $97,813,060$ & 132.79 & 44.5 & 78.0 & 64.2 & 95.6 & 72.7 \\
\hline
\end{tabular}

deletion or duplication in these genes. No deletion or duplication in known genes were found.

In a second approach of finding candidate variants in the family, the exome data of the affected and the unaffected members were analyzed simultaneously. The pedigree was analyzed to establish the most likely inheritance pattern for a shared genetic cause of DDH in the family. De novo occurrence of candidate variants was not considered given the multiple affected family members with similar phenotypes and hence an expected shared genetic cause. X-linked inheritance was considered unlikely as there is an affected female in the family. An autosomal recessive inheritance could not be excluded based on the pedigree. However, homozygosity of a causal variant and compound heterozygosity in both affected family members, cannot be excluded.

Different filter settings were applied to look for potential candidate variants under an autosomal recessive inheritance model. More specifically, the analysis looked for (1) candidate variants present in homozygous state in IV:2, in heterozygous state in III:4, and present in homozygous or heterozygous state in III:5 [assuming at least a partially shared genetic cause of DDH in both affected members], (2) candidate variants present in homozygous state in III:5, in heterozygous state in III:4, and present in homozygous or heterozygous state in IV:2 [assuming at least a partially shared genetic cause of DDH in both affected members] and (3) candidate variants present in compound heterozygous state in IV:2, of which one variant is present in heterozygous state in both III:4 and III:5. In addition, another rare variant in the same gene would then be expected to be present in III:5. However, in accordance with the listed criteria here for an autosomal recessive model, no candidate variants were found.

Finally, an autosomal dominant inheritance with incomplete penetrance was considered. Interestingly, this inheritance pattern has been suggested in scientific literature for familial forms of DDH and is a valid option based on the pedigree. Under this model, individual III:4 (unaffected mother) would be expected to be an asymptomatic carrier of the candidate variant, and the affected individuals III:5 and IV:2 would be expected to both carry this candidate variant.

Similar to the family analysis performed by Feldman et al. [2], only rare variants were taken into account (allele frequencies below or equal to 1\% in 1000G, ExAC and our in-house database of Saudi variants), and only variants located within genes (exonic and intronic) or promoter regions were considered. Furthermore, some quality parameters were taken into account including the depth of coverage $(\mathrm{DP}>10)$ in at least 1 family member, the absence of allelic imbalance in at least 1 family member and good genotype quality $(G Q>20)$ in at least 1 family member.

Of the variants that came out of this filtering procedure, 59 were not present in dbSNP and were, as such, considered as 'new' variants. Genes in which these 59 variants are located are listed in Panel 1 and in supplementary table. Out of 59 variants, variants in 57 genes were present in all family members with no link to hip dysplasia. Only 2 genes (HSPG2 and ATP2B4) were found to be linked to hip dysplasia or other skeletal or bone/joint abnormalities. The variants in these genes include heterozygous rare coding variants in the HSPG2 (c.3328G > T) and ATP2B4 (c.2264G > A) genes.

\section{Sanger validation}

The 59 variants were Sanger sequenced in all three affected individuals (III-5, IV-1, IV-2) and parents (III-3, III4). All three affected individuals shared variants in HSPG2 and $A T P 2 B 4$ genes only. The entire relevant coding exons of the HSPG2 and ATP2B4 genes were subsequently

Table 2 Post-alignment statistics (SNP and INDELS)

\begin{tabular}{|c|c|c|c|c|c|c|c|c|c|c|}
\hline Sample ID & $\begin{array}{l}\text { Number } \\
\text { of SNPs }\end{array}$ & $\begin{array}{l}\text { Synonymous } \\
\text { Variants }\end{array}$ & $\begin{array}{l}\text { Missense } \\
\text { Variants }\end{array}$ & $\begin{array}{l}\text { Stop } \\
\text { Gained }\end{array}$ & $\begin{array}{l}\text { Stop } \\
\text { Lost }\end{array}$ & $\begin{array}{l}\text { Number } \\
\text { of INDELs }\end{array}$ & $\begin{array}{l}\text { Inframe } \\
\text { INDELs }\end{array}$ & $\begin{array}{l}\text { Present in } \\
\text { dbSNP142 (\%) }\end{array}$ & $\begin{array}{l}\text { Het/Homo } \\
\text { Ratio }\end{array}$ & $\begin{array}{l}\text { Ts/TV } \\
\text { Ratio }\end{array}$ \\
\hline|| $\mid-3$ & 48,829 & 9,692 & 8,542 & 67 & 33 & 3,208 & 252 & 98.3 & 1.1 & 2.4 \\
\hline|| $\mid-4$ & 51,488 & 9,632 & 8,457 & 64 & 26 & 3,545 & 237 & 98.1 & 1.0 & 2.4 \\
\hline |II-5 & 56,735 & 10,333 & 9,066 & 79 & 25 & 4,959 & 267 & 97.7 & 1.2 & 2.4 \\
\hline IV-2 & 50,994 & 9,826 & 8,712 & 46 & 28 & 3,343 & 174 & 98.2 & 1.1 & 2.4 \\
\hline
\end{tabular}


sequenced in all affected and unaffected subjects of the family. Variants identified through WES were also detected by Sanger sequencing (Fig. 3). A third affected individual (IV:1) was also screened using Sanger sequencing and was found positive for variants detected by exome sequencing.

\section{In silico analysis}

Variants in HSPG2 and ATP2B4 occur at highly conserved residues (Table 3) and are predicted to be deleterious by the majority of bioinformatics results (Table 4). Moreover, I-Mutant software (used for prediction of protein stability upon single point mutation) predicted the mutant protein

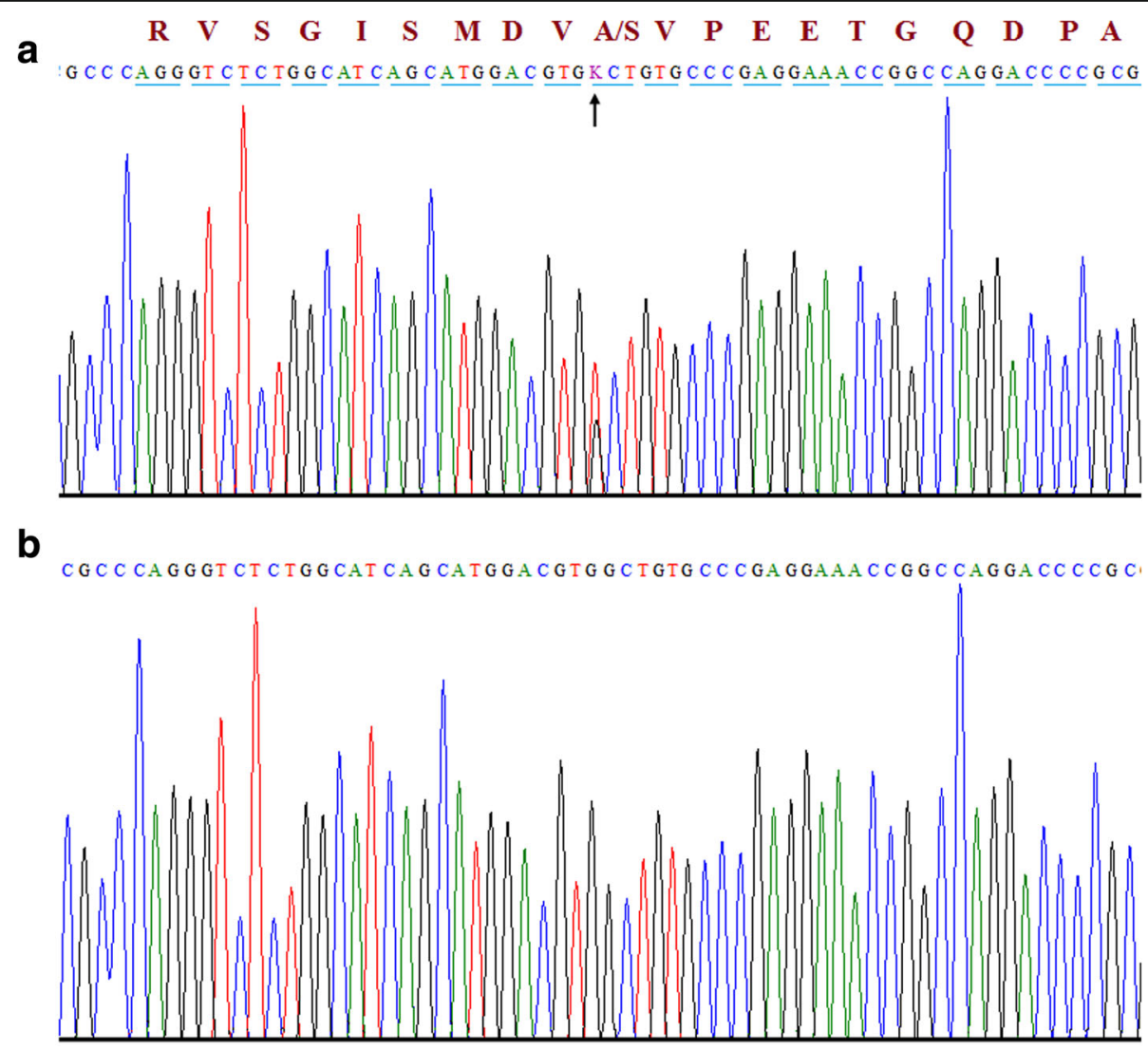

$\begin{array}{lllllllllllllllllllll}C & K & I & W & P & K & L & R & V & L & A & R / Q & S & S & P & T & D & K & H & T\end{array}$ CAAGATCTGG C CTAAG CTI CGGGI C CTGG CG CKAT CTT CT C C CA CTGA CAAG CACA C C C

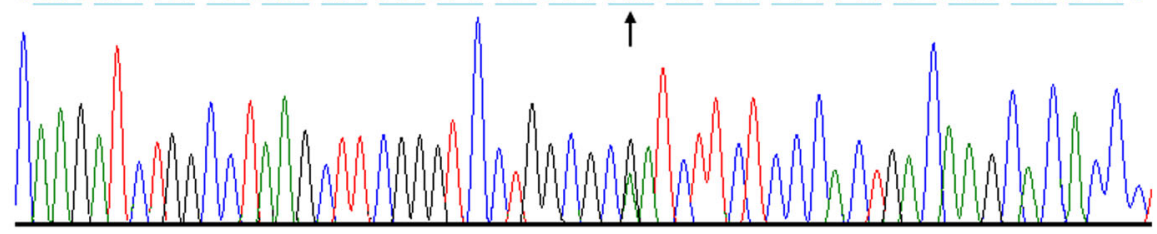

d

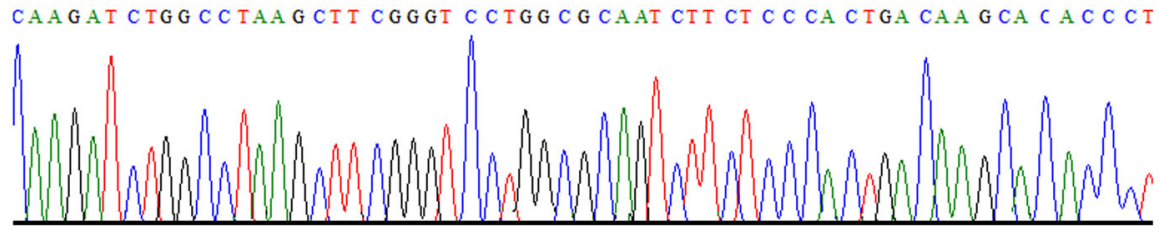

Fig. 3 Sequence chromatograms of heterozygous variants identified in all three individuals of a family segregating DDH. The upper panel (a) represents the nucleotide sequences in all affected individuals in HSPG2 (c.3328G > T) gene and the lower panel (c) represents the nucleotide sequences in all affected individuals in ATP2B4 (c.2264G > A) gene. Panel $\mathbf{b}$ and $\mathbf{d}$ shows partial nucleotide sequence of the control individuals for HSPG2 and ATP2B4. Arrow head indicates the variant site 
Table 3 Multiple sequence alignment for variants in HSPG2 (p. Ala1110Ser) and ATP2B4 (p. Arg755Gln)

\begin{tabular}{lll}
\hline Species & Ensembl ID for HSPG2 & Alignment \\
Human & ENST00000341360 & \\
Mutant & \\
Mmulatta & ENSMMUG00000014828 & \\
Fcatus & ENSFCAG00000002965 & \\
Mmusculus & ENSMUSG00000028763 & Alignment \\
Ggallus & No Homologue & \\
Drerio & ENSDARG00000076564 \\
Xtropicalis & ENSXETG00000017911 \\
Species & Ensembl ID for ATP2B4 \\
Human & ENST00000341360 \\
Mutant & \\
Mmulatta & ENSMMUG00000021116 \\
Fcatus & ENSFCAG00000000870 \\
Mmusculus & ENSMUSG00000026463 \\
Ggallus & ENSGALG00000003601 \\
Drerio & ENSDARG00000044902 \\
Dmelanogaster & FBgn0259214 \\
\hline
\end{tabular}

as less stable [27]. Furthermore, we used DUET (predicting effects of mutations on protein stability via an integrated computational approach), SDM (predicting effects of mutations on protein stability and malfunction) and mCSM (predicting the effect of mutation in protein using graph based signatures) for prediction of effect of mutation on the protein and found that these variants are indeed destabilizing [28-30]. Pathway commons network visualizer ( $\mathrm{PCViz}$ ) was used to detect interaction between HSPG2 and ATP2B4 protein [31]. No direct interaction between these two proteins was detected. However, HSPG2 indirectly controls expression of ATP2B4 via variety of transcription and regulatory factors including GATA1, MAZ and RFX1 (Fig. 4).

\section{Discussion}

Exome variants were filtered considering an autosomal dominant inheritance with incomplete penetrance. Interestingly, this inheritance pattern has been suggested in scientific literature for familial forms of DDH and is a valid option based on the pedigree $[2,7,25]$. This model explains why the mother (III:4) is an asymptomatic carrier of the candidate variant, while the affected individuals IV:2 and III:5 are carrying these candidate variants.

A novel missense variant in HSPG2 was detected in heterozygous state in both affected family members (IV:2 and III:5) and in the unaffected mother (III:4) of IV:2. The unaffected father does not carry this variant. Similarly, a novel missense variant in ATP2B4 was detected in heterozygous state in the affected family members (IV:2 and III:5) and in the unaffected mother (III:4) of IV:2. The unaffected father does not carry this variant. Hence, the segregation of these variants is in accordance with an autosomal dominant inheritance with incomplete penetrance.

HSPG2 encodes for the perlecan protein, a multi domain proteoglycan that cross-links extracellular matrix components and cell-surface molecules, which is essential for many biological activities. It has been shown that perlecan protein is strongly associated with musculoskeletal development, both in mouse and in human, making it a potential candidate gene for skeletal phenotypes such as DDH $[32,33]$. Loss of function mutations in HSPG2 results in a musculoskeletal phenotype in both mouse and humans. For instance, mutations in HSPG2 gene can cause the autosomal recessive conditions Schwartz-Jampel syndrome type 1 (SJS1) and Silverman-Handmaker type of dyssegmental dysplasia. SJS1 is an autosomal recessive disease characterized by permanent myotonia and skeletal dysplasia, resulting in reduced stature, kyphoscoliosis, bowing of the diaphyses and irregular epiphyses. Other symptoms may include eye abnormalities such as cataract, which is also often associated with myotonic dystrophy [34]. Dyssegmental dysplasia Silverman-Handmaker type (DDHS) is an autosomal recessive skeletal dysplasia characterized by marked differences in size and shape of the vertebral bodies (anisospondyly) and short-limbed dwarfism. There are two recognized types: the severe, lethal DDSH (or lethal Kniest-like syndrome) and the milder Rolland-Desbuquois form. Individuals with DDSH also show flat face, micrognathia, cleft palate and reduced joint mobility. Moreover, they have an encephalocoele [35]. Besides these two Mendelian inherited conditions, a missense mutation in HSPG2 (p.Asn786Ser) has recently been linked with idiopathic scoliosis based on exome sequencing analysis of a multigenerational family of European ancestry with familial scoliosis. Interestingly, segregation

Table 4 Rare heterozygous exome variants [HSPG2 (c.3328G > T; p.Ala1110Ser) and ATP2B4 (c.2264G > A; p.Arg755Gln) identified in all affected individuals with developmental dysplasia of the hip

\begin{tabular}{|c|c|c|c|c|c|c|c|c|c|c|c|c|}
\hline Gene & Variant Type & $\begin{array}{l}\text { Global Minor } \\
\text { Allele Frequency }\end{array}$ & PolyPhen2 & SIFT & $\begin{array}{l}\text { Mutation } \\
\text { Taster }\end{array}$ & $\begin{array}{l}\text { Mutation } \\
\text { Assessor }\end{array}$ & CADD & GERP++ & PhastCons & SiPhy & Phylop & VEST3 \\
\hline HSPG2 & Missense & $0.0 \%$ & 0.999 & 0.01 & 1.0 & 2.8 & 26.2 & 5.44 & 0.84 & 10.23 & 0.83 & 0.55 \\
\hline ATP2B4 & Missense & $0.0 \%$ & 0.997 & 0.0 & 1.0 & 4.6 & 35.0 & 5.2 & 1.0 & 18.36 & 0.95 & 0.92 \\
\hline
\end{tabular}

a 1000 Genome, Exome Aggregation Consortium, 64 exome sequences from Saudi individuals 


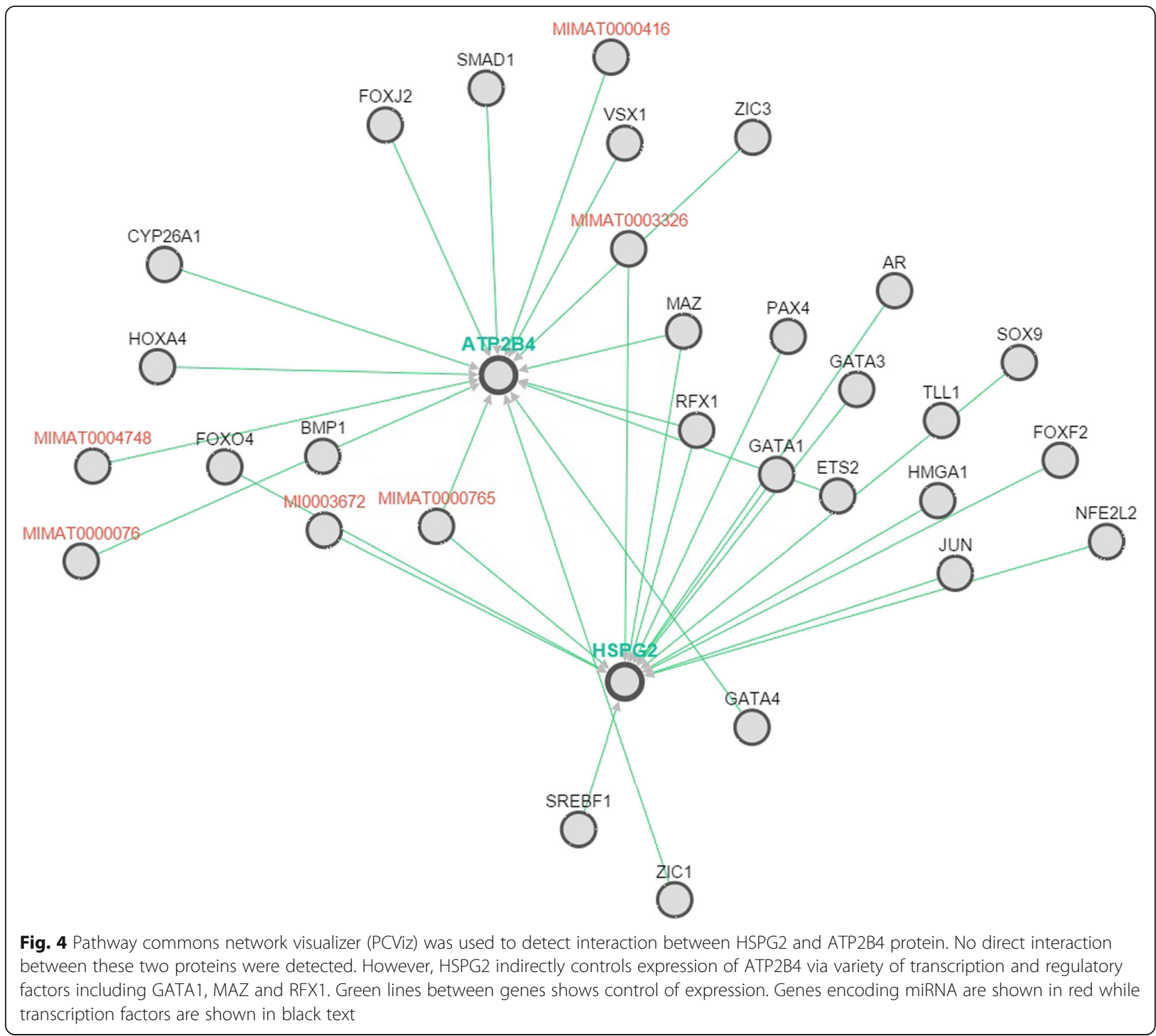

analysis in this family also suggested an autosomal dominant inheritance pattern with incomplete penetrance [36].

The variant detected here causes an alanine to serine (p.Ala1110Ser) missense substitution within laminin IV type A2 domain of the protein, which might affect protein interactions. HSPG2 variation has been linked to hip dysplasia in both human and mice. Hip dysplasia can be present in patients with SJS1 [36]. In mice, a specific mutant HSPG2 strain (C1532Yneo) was described to have reduced body weight and to develop chondrodysplasia and hip dysplasia [37].

$A T P 2 B 4$, also known as PMCA4, encodes the plasma membrane calcium-transporting ATPase 4 enzyme, which is involved in catalyzing the hydrolysis of ATP, coupled with the transport of $\mathrm{Ca}^{2+}$ out of the cell. PCMA proteins including PCMA4 were suggested to play important roles in the regulation of bone homeostasis in both mice and humans by modulating calcium signaling in osteoclasts [38].

Mutations in $A T P 2 B 4$ have only recently been linked to a disease phenotype. More specifically, a specific missense mutation (c.803G > A, p.R268Q) was shown to cause autosomal dominant familial spastic paraplegia in a Chinese family [39]. Further studies on the detected mutation revealed functional changes in calcium homeostasis in human neuronal cells, suggesting that calcium dysregulation may be associated with the phenotype in that family [40].

The variant detected here causes an arginine to glutamine (p.Arg755Gln) missense substitution within a cytoplasmic domain of the ATP2B4 protein.

Based on in silico predictions, the altered residues of HSPG4 and ATP2B4 are conserved (Table 3), and the 
detected changes are predicted to have functional effects at the protein level (Table 4).

\section{Conclusions}

In conclusion, these variants are reported based on their rarity in the general population (absent from 1000G, ExAC and (bSNP), in accordance with an autosomal dominant inheritance with incomplete penetrance shown by the family pedigree and the association of other HSPG2 mutations with several skeletal phenotypes including hip dysplasia in human and mice points towards the role of ATP2B4 in bone formation through modulation of calcium signaling.

A significant enrichment of the detected variants here or other rare HSPG2 and ATP2B4 variants in independent cohorts of DDH patients could provide a further evidence of the role of HSPG4 and ATP2B4 genes in general or the detected variants here in particular in the DDH phenotype.

Pathway analysis identified indirect interaction between HSPG2 and ATP2B4 proteins. HSPG2 regulate the ATP2B4 expression via a variety of transcription factors including GATA1, RFX1 and MAZ (Fig. 4). The genotype-phenotype relationship may be the combined effect of both mutations in each gene. Epistatic relationship between HSPG2 and ATP2B4 genes remain speculative until functional studies are performed.

This study provides the first evidence of a digenic inheritance in DDH in a family and extends the spectrum of genetic heterogeneity in this human DDH. in vitro functional studies are required to understand the mechanism underlying the role of epistatic interactions in DDH.

\section{Additional files}

Additional file 1: Figure S1. Flow chart showing steps performed during generation of annotated variant file from raw data. (JPG 83 kb)

Additional file 2: Figure S2. Variant filtration process illustrating the exome filtering scheme in two affected individuals. Panel 1: Genes containing rare variants (1\% in 1000G/ExAC/in-house database; absent from $\mathrm{dbSNP}$ ) and in accordance with an autosomal dominant inheritance with incomplete penetrance (shared between affected samples III:5 and IV:2). ACOT8, ADCK1, AGMO, ANGPT4, ANKS3, ATP2B4, BARHL1, C12orf44, C18orf56, CACTIN, CCM2L, CEACAM4, CRISPLD2, CTAGE7P, CTSA, CTSE, DAGLB, DAZAP2, DCTN4, DENND1B, EDEM1, EMG1, FAM154A, FAM19A2, GSK3A, HIPK1, HMCN2, HNRNPUL1, HSPG2, INCENP, INTS1, IRG1, KCNIP4, KHSRP, LEPREL2, LPPR3, MAN1C1, MICALCL, MREG, MUC5B, NAV1, NEURL2 NPR2, NUCKS1, PCSK5, PDS5A, PLCG2, POMT1, PPL, PRDM7, PRKAB1, SEZ6L, SLC6A12, SLC7A5, SLIT2, ZCCHC8, ZNF335, ZNF648, ZNF780A. (JPG 128 kb)

\section{Abbreviations}

ATP2B4: ATPase plasma membrane Ca2+ transporting 4; CGID: Center for genetics and inherited diseases; DDH: developmental dysplasia of the hip; HSPG2: heparan sulfate proteoglycan 2; MIM: mendelian inheritance in man; SNP: single nucleotide polymorphism; WES: whole exome sequencing

\section{Acknowledgments}

We are grateful to the patients for giving us permission to carry out the genetic studies and publish their clinical and genetic data in this manuscript. We also thank Prof Mohammed A Hannan for critically reviewing the manuscript.
Funding

No funding source available.

\section{Authors' contributions}

KIK collected samples and carried out clinical evaluation. AMA, EA and SB performed DNA extraction, PCR, genes sequencing and data analysis. SB and KIK drafted the manuscript. All authors have seen and agreed to the content of the manuscript.

Availability of data and materials

DNA samples, vcf files and Sanger reads are available on request

\section{Competing interests}

The authors declare that they have no competing interests.

\section{Consent for publication}

All affected and control individuals signed informed written consent for reporting incidental findings, identifying information and a mutation data underlying the disease.

\section{Ethics approval and consent to participate}

Ethical approval for the study was obtained from the IRB of the Center for Genetics and Inherited Diseases (CGID), Taibah University Almadinah Almunawarah. All affected and control individuals signed informed consent for genetic analysis prior to start of the study.

\section{Publisher's Note}

Springer Nature remains neutral with regard to jurisdictional claims in published maps and institutional affiliations.

\section{Author details}

${ }^{1}$ Centre for Genetics and Inherited Diseases, Taibah University, Almadinah Almunawwarah 30001, Saudi Arabia. ${ }^{2}$ Department of Orthopedic Surgery, College of Medicine, Taibah University, Almadinah Almunawwarah, Saudi Arabia.

Received: 30 October 2016 Accepted: 7 March 2017

Published online: 21 March 2017

References

1. Jacobsen S, Sonne-Holm S, Søballe K, Gebuhr P, Lund B. Hip dysplasia and osteoarthritis: a survey of 4,151 subjects from the Osteoarthritis Substudy of the Copenhagen City Heart Study. Acta Orthop. 2005;76:149-58.

2. Feldman GJ, Parvizi J, Levenstien M, Scott K, Erickson JA, Fortina P, et al. Developmental dysplasia of the hip: linkage mapping and whole exome sequencing identify a shared variant in CX3CR1 in all affected members of a large multigeneration family. J Bone Miner Res. 2013;28:2540-9.

3. Shi D, Dai J, lkegawa S, Jiang Q. Genetic study on developmental dysplasia of the hip. Eur J Clin Invest. 2012;42:1121-5.

4. Cilliers $\mathrm{HJ}$, Beighton P. Beukes familial hip dysplasia: an autosomal dominant entity. Am J Med Genet. 1990;36:386-90.

5. Tonnis D, Heinecke A. Acetabular and femoral anteversion: relationship with osteoarthritis of the hip. J Bone Joint Surg Am. 1999:81:1747-70.

6. Mabuchi A, Nakamura S, Takatori Y, Ikegawa S. Familial osteoarthritis of the hip joint associated with acetabular dysplasia maps to chromosome 13q. Am J Hum Genet. 2006;79:163-8.

7. Ceylaner G, Ceylaner S, Ustünkan F, Inan M. Autosomal dominant inheritance of congenital dislocation of the hip in 16 members of a family. Acta Orthop Traumatol Turc. 2008:42:289-91.

8. Feldman G, Dalsey C, Fertala K, Azimi D, Fortina P, Devoto M, et al. Identification of a $4 \mathrm{Mb}$ Region on Chromosome 17q21 Linked to Developmental Dysplasia of the Hip in One 18-member, Multigeneration Family. Clin Orthop Relat Res. 2010;468:337-44.

9. Woolf $\mathrm{CM}$, Koehn JH, Coleman SS. Congenital hip disease in Utah: the influence of genetic and nongenetic factors. Am J Hum Genet. 1968;20:430-9.

10. Czeizel A, Szentpetery J, Tusnady G, Vizkelety T. Two family studies on congenital dislocation of the hip after early orthopaedic screening Hungary. J Med Genet. 1975;12:125-30.

11. Kramer AA, Berg $K$, Nance WE. Familial aggregation of congenital dislocation of the hip in a Norwegian population. J Clin Epidemiol. 1988;41:91-6. 
12. Wilkinson JA. Etiologic factors in congenital displacement of the hip and myelodysplasia. Clin Orthop Relat Res. 1992;281:75-83.

13. Basit, S., Hannan, M. A. \& Khoshhal, K. I. Developmental dysplasia of the hip: Usefulness of next generation genomic tools for characterizing the underlying genes; a mini review. Clin Genet. 2016. doi: 10.1111/cge.12755

14. Karapinar L, Surenkok F, Oztürk H, Us MR, Yurdakul L. The importance of predicted risk factors in developmental hip dysplasia: an ultrasonographic screening program. Acta Orthop Traumatol Turc. 2002;36:106-10

15. Bialik V, Bialik GM, Blazer S, Sujov P, Wiener F, Berant M. Developmental dysplasia of the hip: a new approach to incidence. Pediatrics. 1999;103:93-9.

16. Rubini M, Cavallaro A, Calzolari E, Bighetti G, Sollazzo V. Exclusion of COL2A1 and VDR as developmental dysplasia of the hip genes. Clin Orthop Relat Res. 2008;466:878-83.

17. Mirdad T. Incidence and pattern of congenital dislocation of the hip in Aseer region of Saudi Arabia. West Afr J Med. 2002;21:218-22.

18. Kremli MK, Alshahid AH, Khoshhal Kl, Zamzam MM. The pattern of developmental dysplasia of the hip. Saudi Med J. 2003;24:1118-20.

19. Moosa NK, Kumar PT, Mahmoodi SM. Incidence of developmental dysplasia of the hip in Dubai. Saudi Med J. 2009:30:952-5.

20. Li LY, Sun XK, Zhao Q, Zhang LJ, Li QW, Wang LL, Gao H. Gene mapping of developmental dysplasia of the hip in chromosome 17q21 region. Zhonghua Yi Xue Yi Chuan Xue Za Zhi. 2010;27:620-5.

21. Dai J, Shi D, Zhu P, Qin J, Ni H, Xu Y, et al. Association of a single nucleotide polymorphism in growth differentiate factor 5 with congenital dysplasia of the hip: a case-control study. Arthritis Res Ther. 2008;10:R126.

22. Shi D, Shi D, Zhu P, Dai J, Zhu L, Zhu H, et al. Association of a single nucleotide polymorphism in Tbx4 with developmental dysplasia of the hip: a case-control study. Osteoarthritis Cartilage. 2010;18:1592-5.

23. Shi D, Dai J, Zhu P, Qin J, Zhu L, Zhu H, et al. Association of the D repeat polymorphism in the ASPN gene with developmental dysplasia of the hip: a case-control study in Han Chinese. Arthritis Res Ther. 2011;13:R27.

24. Kolundžic', R., Trkulja, V., Mikolaučric', M., Kolundžić, M. J., Pavelić, S. K. \& Pavelić K. Association of interleukin-6 and transforming growth factor-b1 gene polymorphisms with developmental hip dysplasia and severe adult hip osteoarthritis: a preliminary study. Cytokine. 54, 125-128 (2011).

25. Watson CM, Crinnion LA, Gleghorn L, Newman WG, Ramesar R, Beighton P, et al. Identification of a mutation in the ubiquitin-fold modifier 1-specific peptidase 2 gene, UFSP2, in an extended South African family with Beukes hip dysplasia. S Afr Med J. 2015:105:558-63.

26. Purcell S, Neale B, Todd-Brown K, Thomas L, Ferreira MA, Bender D, et al. PLINK: a tool set for whole-genome association and population-based linkage analyses. Am J Hum Genet. 2007;81:559-75.

27. Capriotti, E., Fariselli, P., Casadio, R. I-Mutant2.0: predicting stability changes upon mutation from the protein sequence or structure. Nucleic. Acids. Res 33(Web Server issue), W306-10 (2005).

28. Worth, C. L., Preissner, R., Blundell, T. L. SDM-a server for predicting effects of mutations on protein stability and malfunction. Nucleic. Acids. Res. 39(Web Server issue), W215-22 (2011).

29. Pires, D. E., Ascher, D. B., Blundell, T. L. DUET: a server for predicting effects of mutations on protein stability using an integrated computational approach. Nucleic. Acids. Res. 42(Web Server issue), W314-19 (2014).

30. Pires DE, Ascher DB, Blundell TL. mCSM: predicting the effects of mutations in proteins using graph-based signatures. Bioinformatics. 2014;30:335-42.

31. Cerami EG, Gross BE, Demir E, Rodchenkov I, Babur O, Anwar N, et al. Pathway Commons, a web resource for biological pathway data. Nucleic Acids Res. 2011;39(Database issue):D685-690.

32. Baschal, E. E., Wethey, C. I., Swindle, K., Baschal, R. M., Gowan, K., Tang, N. L., et al. Exome sequencing identifies a rare HSPG2 variant associated with familial idiopathic scoliosis. G3 (Bethesda). 5, 167-174 (2014).

33. Lowe, D. A., Lepori-Bui, N., Fomin, P. V., Sloofman, L, G., Zhou, X., FarachCarson, M. C. et al. Deficiency in perlecan/HSPG2 during bone development enhances osteogenesis and decreases quality of adult bone in mice. Calcif. Tissue. Int. 95, 29-38 (2014).

34. Nicole S, Davoine CS, Topaloglu H, Cattolico L, Barral D, Beighton P, et al. Perlecan, the major proteoglycan of basement membranes, is altered in patients with Schwartz-Jampel syndrome (chondrodystrophicmyotonia). Nat Genet. 2000:26:480-3.

35. Arikawa-Hirasawa E, Wilcox WR, Le AH, Silverman N, Govindraj P, Hassell $J R$, et al. Dyssegmental dysplasia, Silverman-Handmaker type, is caused by functional null mutations of the perlecan gene. Nat Genet 2001:27:431-4

36. Stum M, Davoine CS, Vicart S, Guillot-Noël L, Topaloglu H, Carod-Artal FJ, et al. Spectrum of HSPG2 (Perlecan) mutations in patients with SchwartzJampel syndrome. Hum Mutat. 2006;27:1082-91.

37. Stum M, Girard E, Bangratz M, Bernard V, Herbin M, Vignaud A, et al. Evidence of a dosage effect and a physiological endplate acetylcholinesterase deficiency in the first mouse models mimicking Schwartz-Jampel syndrome neuromyotonia. Hum Mol Genet. 2008;17:3166-79.

38. Kim HJ, Prasad V, Hyung SW, Lee ZH, Lee SW, Bhargava A, et al. Plasma membrane calcium ATPase regulates bone mass by fine-tuning osteoclast differentiation and survival. J Cell Biol. 2012;199:1145-58.

39. Li M, Ho PW, Pang SY, Tse ZH, Kung MH, Sham PC, et al. PMCA4 (ATP2B4) mutation in familial spastic paraplegia. PLoS One. 2014;9, e104790.

40. Ho PW, Pang SY, Li M, Tse, Z. H., Kung, M. H., Sham, P. C. et al. PMCA4 (ATP2B4) mutation in familial spastic paraplegia causes delay in intracellular calcium extrusion. Brain. Behav. 5, e00321 (2015).

\section{Submit your next manuscript to BioMed Central and we will help you at every step:}

- We accept pre-submission inquiries

- Our selector tool helps you to find the most relevant journal

- We provide round the clock customer support

- Convenient online submission

- Thorough peer review

- Inclusion in PubMed and all major indexing services

- Maximum visibility for your research

Submit your manuscript at www.biomedcentral.com/submit
) Biomed Central 